\title{
Cationic species as dopants for organic semiconductors
}

\begin{abstract}
Herein a short review of cationic species reported as dopants for organic semiconductors is presented. The approach suggests using the same cationic species for both $\mathrm{p}$ - and n-type doping of organic matrices with organic dopants. As an example, pyronin B chloride and benzyl viologen dichloride are reviewed as universal dopants working both ways. Different device characteristics and measurements confirm the donor and acceptor properties of these organic molecules.
\end{abstract}

Keywords: doping, organic semiconductors, n-type dopant, p-type dopant, benzyl viologen dichloride, organic field-effect transistors, conjugated polymers

\section{Introduction}

Organic semiconductors (OSCs) are the potential key materials for future flexible electronics due to their outstanding mechanical and optoelectronic properties ${ }^{1,2}$ Electronic devices such as organic light emitting diodes have already shown successful progress for flat panel displays, and application of OSCs is gradually expanding to various fields of electronics. ${ }^{1-3}$ However, OSCs still lag behind inorganic ones due to their poor electrical properties including low charge carrier mobility and conductivity as well as device stability issues. ${ }^{4-7}$ In order to overcome these issues, several approaches have been developed one of which is doping. ${ }^{5}$ Doping is one of the most effective methods to improve electrical properties of OSCs through increasing their charge carrier density and mobility. ${ }^{2,8-11}$ However, the doping of OSCs is different from the doping of inorganic ones. Unlike the latter, doping in organic electronics does not assume the replacement of a host lattice atom by an impurity atom. It is rather a simple charge transfer between two molecules., ${ }^{2,8,9,12-14}$ A significant number of research groups have reported different kinds of dopants for OSCs. In this review, a summary of the dopants, namely cationic species that can be applied as both p- and n-type dopants, is introduced.

a)
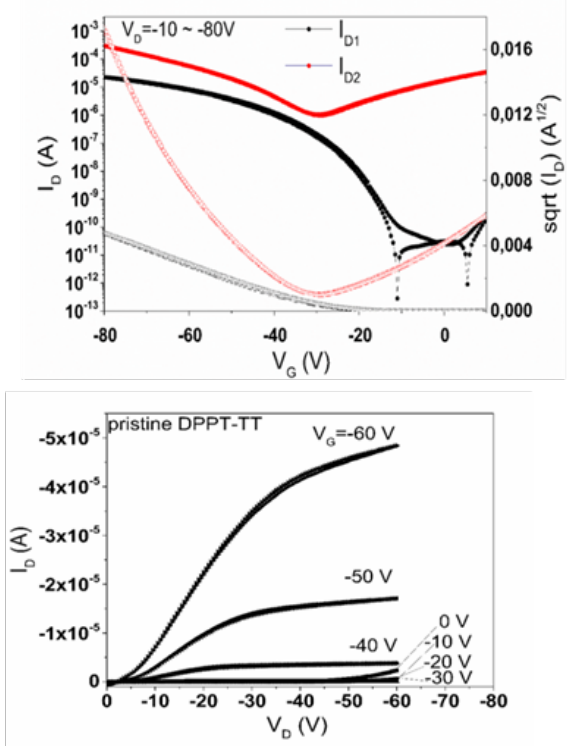

Volume 3 Issue 2 - 2019

\author{
Gunel Huseynov \\ Dongguk University, South Korea
}

Correspondence: Huseynova G, Dongguk University, Pildongro 30, Jung-gu, Seoul, South Korea, Tel +82 I0 3064-5407, Emailbblg7839@gmail.com

Received: December 26, 2018 | Published: March 29, 2019

\section{Cationic dyes as dopants for organic molecules}

\section{Pyronin B}

As the first example, pyronin B dichloride (PyB) is given. It is a solution processed cationic non-polymeric dye. PyB belongs to xanthene dyes, which are salts of organic bases. They are called cationic dyes, because their molecules ionize in solution, causing the colored component to become a positively charged radical which reacts with the anionic sites of the other molecules they are blended with. Accordingly, they act as an electron deficient site, which makes them perfect p-type dopants. PyB was reported as a p-dopant for diketopyrrolopyrrole-thieno [3,2-b]thiophene (DPPT-TT), an ambipolar conjugated copolymer, in a simple solution-processed doping. ${ }^{15}$ The doped polymer was used as an active layer for OFETs. As a result, device performance including hole mobility and threshold voltage was significantly improved (Figure 1). Pyronin B was also reported as an effective and stable n-type dopant when reduced to its neutral leuco (colorless) state through both solid-state ad solutionprocessed doping. ${ }^{16-18}$

b)
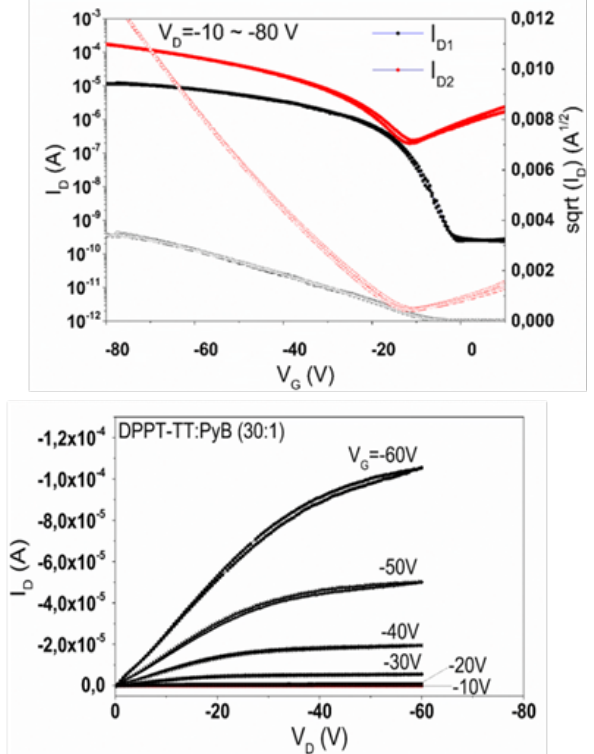

Figure I Representative transfer and output curves for DPPT-TT OFETs: (A) un doped; (B) PyB doped (30:I). $I_{D}, V_{D}, V_{G}-$ drain current, drain voltage and gate voltage, respectively. 


\section{Viologens}

Another cationic compound, benzyl viologen dichloride (BVD) was recently reported as an effective n-type dopant for OSCs when reduced to its neutral state. BVD consists of two chlorine anions and a positively charged organic molecule with two nitrogen cations. BVD was used as the n-type dopant, since it exhibits strong electron donating properties originating from the viologen unit due to low reduction potentials and redox-rich nature. All viologen compounds, including benzyl viologen, typically undergo three reduction states in redox reactions, forming different colored compounds in solution. Their initial energetically stable cation $\left(\mathrm{V}^{2+}\right)$ is colorless, whereas the single electron reduced form (radical mono cation, $\mathrm{V}^{+}$) is intensely violet, and the third reduced state (neutral $\mathrm{V}^{0}$ ) is yellow. The last two states both readily transfer their electrons but the neutral state is the most reactive, and a stronger reducing agent than the mono cations due to the lower reduction potential. Benzyl viologen has the lowest reduction potential among all viologens. Benzyl viologen reduction potentials are in the range from -0.33 to $-0.79 \mathrm{~V}$ for the $\mathrm{V}^{2+}$, and $\mathrm{V}^{0}$ and $\mathrm{V}^{+}$states, respectively. To use BVD for doping purposes, it was reduced chemically to obtain the electron donating neutral state from the di-cation form. As a result, the polymers doped with BVD exhibited high n-channel performance when applied to OFETs (Figure 2).

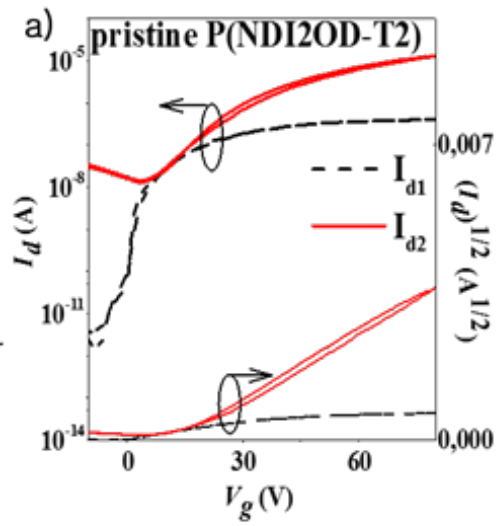

d)

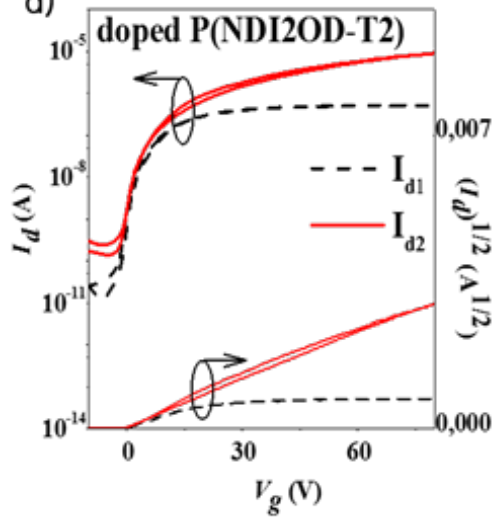

b)

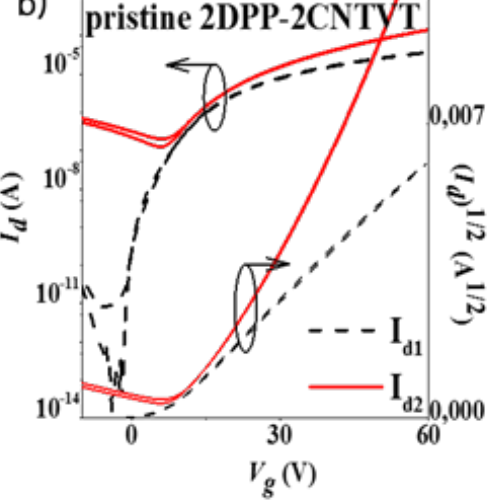

e)

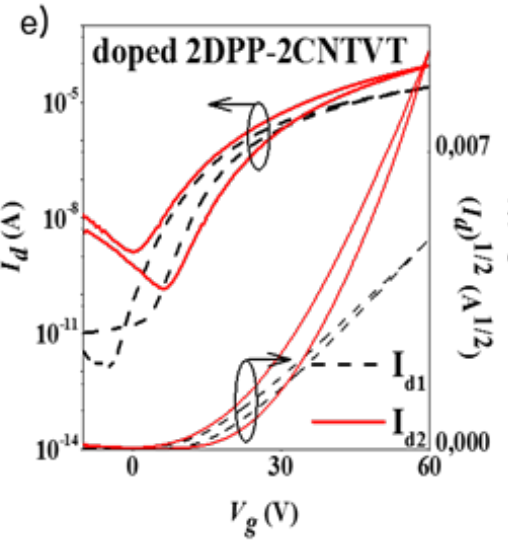

c)

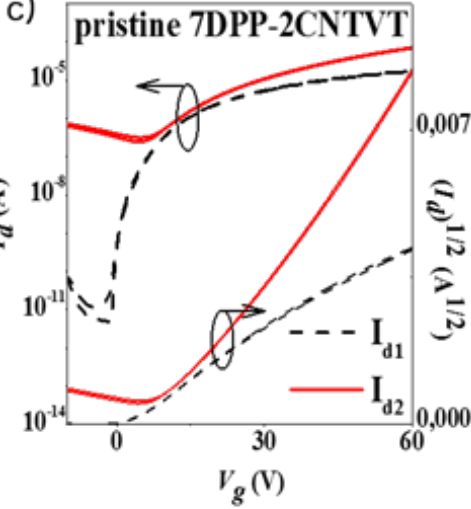

f)

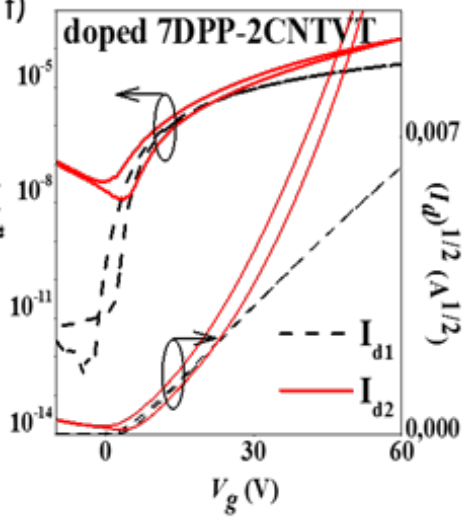

Figure 2 Transfer characteristics for pristine and doped (A) and (D) P(NDI2OD-T2), (B) and (E) 2DPP-2CNTVT, and (C) and (F) 7DPP-2CNTVT OFETs, respectively. $I_{d 1}$ and $I_{d 2}$ are the drain currents in linear $\left(V_{d}=10 \mathrm{~V}\right)$ and saturation regimes $\left(V_{d}=60\right.$ and 80V for 2DPP-2CNTVT and 7DPP-2CNTVT, and $\mathrm{P}\left(\right.$ NDI2OD-T2) based devices), respectively. $I_{\phi} V_{\phi}$ and $V_{g}$ stand for the drain current, drain voltage, and gate voltage, respectively.

\section{Conclusion}

This article reviewed $\mathrm{n}$ - and $\mathrm{p}$-type doping effects of two cationic compounds, PyB and BVD for various organic semiconductors. These two examples of cationic species have demonstrated that the same cationic compound can be effectively used as both p-type dopant when used as is and n-type dopant when reduced to their neutral states. The current study suggests a new class of universal dopants for organic electronics and will inspire follow-up approaches in this field.

\section{Acknowledgments}

None.

\section{Conflicts of interest}

Authors declare that there is no conflict of interest.

\section{References}

1. Facchetti, A. $\pi$-Conjugated Polymers for Organic Electronics and Photovoltaics Cell Applications. Chem Mater. 2011, 23, 733-758.

2. Walzer K, Maennig B, Pfeiffer M, et al. Highly Efficient Organic Devices Based on Electrically Doped Transport Layers. Chem Rev. 2007; 107:1233-1271.

3. Geffroy B, Le Roy P, Prat C. Organic Light-Emitting Diode (OLED) 
Technology: Materials, Devices and Display Technologies. Polym Int. 2006;55:572-582

4. Baumeier B,May F, Lennartz C, et al. Challenges for in Silico Design of Organic Semiconductors. J Mater Chem. 2012;22:10971.

5. Loo YL, McCulloch I. Progress and Challenges in Commercialization of Organic Electronics. MRS Bulletin. 2008;33:653.

6. Coropceanu V, Cornil J, Da Silva Filho DA, et al. Charge Transport in Organic Semiconductors. Chem Rev. 2007;107:926-952.

7. Zhou TC, Chen G, Liao RJ, et al. Charge Trapping and Detrapping in Polymeric Materials: Trapping Parameters. $J$ Appl Phys. 2011;110:043724

8. Lüssem B, Riede M, Leo K. Doping of Organic Semiconductors. Phys Status Solidi A. 2013;210(1).

9. Salzmann I, Heimel G, Oehzelt M, et al. Molecular Electrical Doping of Organic Semiconductors: Fundamental Mechanisms and Emerging Dopant Design Rules. Acc Chem Res. 2016;49:370-378.

10. Long DX, Xu Y, Wei HX, et al. Controlling Charge Injection Properties in Polymer Field-Effect Transistor by Incorporation of Solution Processed Molybdenum Trioxide. Phys Chem Chem Phys. 2015;17:20160.

11. Nugraha M, Kumagai I, Watanabe S, et al. Enabling Ambipolar to Heavy n-Type Transport in PbS Quantum Dot Solids through Doping with Organic Molecules. ACS Appl Mater Interfaces. 2017;9:18039-18045.
12. Jacobs IE, Moule AJ. Controlling Molecular Doping in Organic Semiconductors. Adv Mater. 2017;29:1703063.

13. Lussem B, Keum CM, Kasemann D, et al. Doped Organic Semiconductors. Chem Rev. 2016;116:13714-13751.

14. Tietze ML, Pahner P, Schmidt K, et al. Doped Organic Semiconductors: Trap-Filling, Impurity Saturation, and Reserve Regimes. Adv Funct Mater. 2015;25:2701-2707.

15. Huseynova $\mathrm{G}, \mathrm{Xu} \mathrm{Y}$, Yawson $\mathrm{BN}$, et al. P-Type Doped Ambipolar Polymer Transistors by Direct Charge Transfer from a Cationic Dye Pyronin B Ferric Chloride. Organic Electronics. 2016;39:229-235.

16. Werner AG, Li F, Harada K, et al. Pyronin B as a Donor for N-Type Doping of Organic Thin Films. Appl Phys Lett. 2003;82:4495.

17. Lee EK, Kim Y, Back J, et al. Reduced Pyronin B as a SolutionProcessable and Heating-Free N-Type Dopant for Soft Electronics. Mater Chem C. 2018;6:6672-6679.

18. Huseynova G, Shin EY, Park WT, et al. Lithium Benzoate doped High Performance n-type Diketopyrrolopyrrole based Organic Thin-Film Transistors. Dyes and Pigments. 2019;162:243-248. 